\title{
General practice
}

\section{Using thresholds based on risk of cardiovascular disease to target treatment for hypertension: modelling events averted and number treated}

\author{
Simon Baker, Patricia Priest, Rod Jackson
}

Editorial by Jackson
Health Funding
Authority, Private
Bag 92522,
Wellesley St,
Auckland, New
Zealand
Simon Baker
registrar in public
health medicine
Wolfson College,
Oxford OX2 6UD
Patricia Priest
Nuffield fellow
Department of
Community Health,
Faculty of Medical
and Health Science,
University of
Auckland, Private
Bag 92019,
Auckland,
New Zealand
Rod Jackson
professor of
epidemiology
Correspondence to:
R Jackson
rt.jackson@
auckland.co.nz

BMJ 2000;320:680-5

\begin{abstract}
Objective To estimate the impact of using thresholds based on absolute risk of cardiovascular disease to target drug treatment to lower blood pressure in the community.

Design Modelling of three thresholds of treatment for hypertension based on the absolute risk of cardiovascular disease. 5 year risk of disease was estimated for each participant using an equation to predict risk. Net predicted impact of the thresholds on the number of people treated and the number of disease events averted over 5 years was calculated assuming a relative treatment benefit of one quarter.

Setting Auckland, New Zealand.

Participants 2158 men and women aged 35-79 years randomly sampled from the general electoral rolls. Main outcome measures Predicted 5 year risk of cardiovascular disease event, estimated number of people for whom treatment would be recommended, and disease events averted over 5 years at different treatment thresholds.

Results 46374 (12\%) Auckland residents aged 35-79 receive drug treatment to lower their blood pressure, averting an estimated 1689 disease events over 5 years. Restricting treatment to individuals with blood pressure $\geqslant 170 / 100 \mathrm{~mm} \mathrm{Hg}$ and those with blood pressure between 150/90-169/99 $\mathrm{mm} \mathrm{Hg}$ who have a predicted 5 year risk of disease $\geqslant 10 \%$ would increase the net number for whom treatment would be recommended by 19401 . This $42 \%$ relative increase is predicted to avert 1139/1689 (68\%) additional disease events overall over 5 years compared with current treatment. If the threshold for 5 year risk of disease is set at $15 \%$ the number recommended for treatment increases by $<10 \%$ but about $620 / 1689$ (37\%) additional events can be averted. A 20\% threshold decreases the net number of patients recommended for treatment by about $10 \%$ but averts 204/1689 (12\%) more disease events than current treatment.

Conclusions Implementing treatment guidelines that use treatment thresholds based on absolute risk could significantly improve the efficiency of drug treatment to lower blood pressure in primary care.
\end{abstract}

\section{Introduction}

Numerous randomised trials have shown that drug treatment for hypertension in patients with blood pressure higher than about $150 / 90 \mathrm{~mm} \mathrm{Hg}$ reduces the relative risk of cardiovascular disease by about one quarter to one third, regardless of blood pressure before treatment or the absolute risk of cardiovascular disease before treatment. ${ }^{1}$ However, the absolute reduction in the risk of cardiovascular disease is directly proportional to the absolute risk of cardiovascular disease before treatment (that is, the incidence of cardiovascular disease). ${ }^{2}$ Most guidelines for treating hypertension therefore recommend that patients with a high absolute risk be treated as a priority. ${ }^{3-6} \mathrm{~A}$ patient's absolute risk of cardiovascular disease depends on the combined effect of their risk factors particularly whether there is a history of symptomatic cardiovascular disease, diabetes, or target organ damage, and whether the patient is older, male, smokes, has dyslipidaemia, and has high blood pressure. $^{7}$

Since 1992, guidelines from New Zealand, both for the management of raised blood pressure ${ }^{38}$ and dyslipidaemia, ${ }^{9}{ }^{10}$ have provided a tool, based on the Framingham heart study, to help practitioners to assess quantitatively the risk of cardiovascular disease in individual patients. The New Zealand guidelines for the management of mildly raised blood pressure (that is, between about 150/90 and 169/99 $\mathrm{mm} \mathrm{Hg}$ ) recommend that the decision to treat should be influenced by the predicted absolute risk of cardiovascular disease; the guidelines suggest that a 5 year risk of $10 \%$ is a reasonable starting point for discussing drug treatment with patients whose blood pressure is mildly raised. ${ }^{3}$ Recent guidelines from the British Hypertension Society have made similar recommendations. ${ }^{4}$ However, the appropriate threshold of the absolute risk of cardiovascular disease at which drug treatment should be initiated is somewhat arbitrary and is based more on historical practices than explicit estimation of the impact on individuals or populations. We attempt to quantify the implications for a population resulting from the use of different treatment thresholds based on the absolute risk of disease. We estimated the number of patients for whom treatment would be recommended and the likely 
number of cardiovascular disease events averted in a well defined urban population.

\section{Participants and methods}

\section{Study population and data collection}

Participants were recruited from the University of Auckland heart and health study, a prevalence survey of risk factors carried out in Auckland, New Zealand, in 1993-4. The study methodology has been described elsewhere. ${ }^{11}$ Briefly, about 2500 residents of Auckland aged 35-84 years were identified by age stratified random sampling of the general electoral rolls (which are over $95 \%$ complete for people of European origin); they were invited to attend a free clinic to assess their risk factors for cardiovascular disease. Trained interviewers administered a structured questionnaire, and, after participants had been seated for five minutes, systolic and diastolic blood pressures were measured twice to the nearest $2 \mathrm{~mm} \mathrm{Hg}$ using a Hawksley random zero sphygmomanometer. A non-fasting blood sample was also drawn. Total cholesterol concentrations were analysed using the method of Allain et al, and concentrations of high density lipoprotein cholesterol were measured using a modification of the method of Lopes-Virella. ${ }^{12}{ }^{13}$

The general electoral roll sampling frame did not include a representative sample of Maori people because about half of all Maori are registered on separate electoral rolls. The general electoral rolls also significantly underrepresent the true proportion of Pacific Islanders in the general population. Because Maori and Pacific Islanders identified from the general rolls were unlikely to be representative, they were excluded from the analysis. The study population of 2158 participants included $2106(97.6 \%)$ people of European origin, 28 (1.3\%) of Chinese origin, $9(0.4 \%)$ people of Indian (subcontinent) origin, and $14(0.6 \%)$ who were classed as belonging to other races.

\section{Estimating cardiovascular risk and treatment benefit}

A published equation for predicting the incident risk of cardiovascular disease from the Framingham heart study was used to calculate each participant's 5 year risk of a fatal cardiovascular disease event or a first disease event that was not fatal. ${ }^{7}$ The independent variables included in the equation were age, sex, systolic blood pressure, ratio of total cholesterol to high density lipoprotein cholesterol, smoking status, and diabetes status. A variable identifying individuals with left ventricular hypertrophy detected by electrocardiography was set to absent for all participants since this risk factor is uncommon and was not assessed. A cardiovascular disease event was defined as one of the following: new onset of angina, myocardial infarction (including silent myocardial infarction diagnosed by electrocardiography), death from coronary heart disease, stroke or transient ischaemic attack, congestive heart failure, or peripheral vascular disease.

The pretreatment blood pressure of participants taking antihypertensive drugs was estimated by adding $12 \mathrm{~mm} \mathrm{Hg}$ to the systolic pressure and $6 \mathrm{~mm} \mathrm{Hg}$ to the diastolic pressure; this adjustment was based on the average treatment effect described in randomised controlled trials. ${ }^{2}$ Participants with a history of cardiovas- cular disease and a predicted 5 year risk of disease estimated at $<20 \%$ were assigned a 5 year risk of $20 \%$. This is based on a minimum rate of events of about $20 \%$ found in control groups in trials of the secondary prevention of cardiovascular disease. ${ }^{14-16}$ Those with previous cardiovascular disease and a predicted 5 year risk of disease $>20 \%$ were classed according to the prediction equation.

Participants were then categorised according to whether treatment would be recommended under the 1995 New Zealand hypertension guidelines using their blood pressure and predicted 5 year risk of cardiovascular disease. ${ }^{3}$ Although these guidelines recommend a threshold of 5 year risk of $10 \%$, we applied three risk thresholds to each participant. Firstly, participants with a systolic blood pressure $\geqslant 170 \mathrm{~mm} \mathrm{Hg}$ or a diastolic pressure $\geqslant 100 \mathrm{~mm} \mathrm{Hg}$ were assigned to the treatment group irrespective of their predicted 5 year risk because there is a strong international consensus that this group should be treated. Secondly, those with systolic pressure $<150 \mathrm{~mm} \mathrm{Hg}$ and diastolic $<90 \mathrm{~mm}$ $\mathrm{Hg}$ were assigned to the non-treatment group irrespective of their predicted 5 year risk, apart from those already receiving drug treatment to lower blood pressure and with a 5 year risk above the thresholds used in the analyses (see below). This exception was made since it was almost certain that their blood pressure before treatment would have been $>150 \mathrm{~mm} \mathrm{Hg}$ systolic or $>90 \mathrm{~mm} \mathrm{Hg}$ diastolic because no treatment guidelines in New Zealand before 1995 recommended antihypertensive treatment for patients with blood pressures below these values. Thirdly, all other participants were assigned to treatment or non-treatment groups in three different analyses using 5 year thresholds for risk of cardiovascular disease of $10 \%, 15 \%$, and $20 \%$. The mean predicted individual 5 year risk of cardiovascular disease in each of the three age groups (35-49, 50-64, and 65-79) was used to predict the number of disease events expected to occur over the next 5 years. The number for whom treatment would be recommended and the number of disease events caused (by stopping treatment in low risk patients) or averted (by initiating treatment in high risk patients) at each risk threshold were calculated by assuming that treatment reduces the incidence of cardiovascular disease by one quarter. All calculations were done using Microsoft Excel spreadsheets and Statistical Analysis System software.

\section{Results}

\section{Participants}

Included in the analysis were 2158 non-Maori and non-Pacific Islander participants from the Auckland University Heart and Health Study who were aged $35-79$ years and for whom data were complete $(72 \%$ of the 3000 invited). Women accounted for $49 \%$ (1064) of the participants.

The distribution of the use of antihypertensive drugs by blood pressure (corrected for treatment effect), age group, and sex is shown in table 1. Blood pressure and the use of antihypertensive drugs increased with age in both sexes. The proportions receiving treatment increased from a few per cent among those aged 35-49 to one third in women aged 65-79. The proportions with higher blood pressure 
Table 1 Blood pressure in study participants (corrected for treatment effect). ${ }^{*}$ Values are numbers (percentages)

\begin{tabular}{|c|c|c|c|}
\hline & & Age (years) & \\
\hline & $35-49$ & $50-64$ & $65-79$ \\
\hline Men & & & \\
\hline No treatment $(\mathrm{n}=928)$ & 320 & 325 & 283 \\
\hline Blood pressure: & & & \\
\hline Group 1 & 294 (92) & $263(81)$ & $195(69)$ \\
\hline Group 2 & $19(6)$ & $55(17)$ & $71(25)$ \\
\hline Group 3 & 7 (2) & 7 (2) & $17(6)$ \\
\hline Treatment† $(n=166)$ & $14 / 334(4)$ & 49/374 (13) & $103 / 386(26.7)$ \\
\hline Blood pressure: & & & \\
\hline Group 1 & $13(93)$ & $26(53)$ & $43(42)$ \\
\hline Group 2 & $1(7)$ & $21(43)$ & $40(39)$ \\
\hline Group 3 & 0 & $2(4)$ & $20(19)$ \\
\hline Women & & & \\
\hline No treatment $(\mathrm{n}=966)$ & 315 & 293 & 258 \\
\hline Blood pressure: & & & \\
\hline Group 1 & 309 (98) & $240(82)$ & $168(65)$ \\
\hline Group 2 & $6(2)$ & $41(14)$ & $72(28)$ \\
\hline Group 3 & 0 & $12(4)$ & $18(7)$ \\
\hline Treatment† $(n=198)$ & $7 / 316$ (2) & $60 / 300(17)$ & $131 / 389(34)$ \\
\hline Blood pressure: & & & \\
\hline Group 1 & $6(86)$ & $43(71)$ & $62(47)$ \\
\hline Group 2 & $1(14)$ & $13(22)$ & $52(40)$ \\
\hline Group 3 & (0) & $4(7)$ & 17 (13) \\
\hline
\end{tabular}

that was not treated also increased with age. Moreover, the blood pressure of older patients who were being treated was higher than that of younger people who were being treated.

The distribution of the predicted 5 year risk of cardiovascular disease by age group, sex, and treatment is shown in table 2 . The mean predicted risk of disease increased markedly with age in both sexes, irrespective of whether participants were being treated. The risk in men was considerably higher than in women at all ages in both treated and untreated groups. Both men and women who were being treated had higher mean predicted risks of disease than those who were not being treated; the difference between the treated and untreated groups was greater among women. In the youngest age group the majority of those receiving treatment were predicted to be at low risk of disease.

\section{Number treated versus events averted}

By extrapolating from the sample to the population of Auckland (390 492 people aged 35-79 years according to the 1991 census), we estimated that about 27368 disease events would occur over 5 years if no one was treated with antihypertensive drugs (table 3). Half of these events were predicted to occur in people aged 65-79 years and significantly more disease events were predicted to occur among men (16 500) than women (10 500) (data not shown).

Altogether, 46374 Auckland residents (20 500 men) aged 35-79 were estimated to be receiving drug treatment to lower their blood pressure. This treatment was estimated to have averted almost 1700 disease events over a 5 year period, with similar numbers of events averted in men and women. Over half of the estimated events averted (1116) were among those aged 65-79.
Table 3 also shows the estimated number of Auckland residents aged 35-79 for whom drug treatment would be recommended and the predicted impact that treatment using each of the three thresholds would have on rates of disease events.

If a 5 year risk of cardiovascular disease of $10 \%$ is used as the threshold for offering treatment and is applied to those aged 35-79, about 13 221 patients currently receiving drug treatment would not meet the criteria for treatment; however, treatment would be recommended for 32662 untreated patients. If a relative benefit of treatment of $25 \%$ is assumed, the predicted number of disease events occurring over 5 years would increase by about 225 among those for whom treatment would no longer be recommended but the threshold would avert an additional 1364 events among those starting treatment. The net additional number of events averted in 5 years is 1139 (800 in men), and represents a 68\% improvement over current treatment at a net cost of a $42 \%$ increase in the number treated (about 19401 new patients; 14500 men). The greatest increase in the number treated would occur among 65-79 year olds (about 14645 ). This group was also predicted to have the greatest reduction in disease events (a decrease of around 800).

If a 5 year risk of $15 \%$ is used as the treatment threshold, about 20339 patients currently receiving drug treatment would no longer meet criteria for treat-

Table 2 Five year risk of cardiovascular disease among participants. Values are numbers (percentages)

\begin{tabular}{lrrr} 
& \multicolumn{3}{c}{ Age (years) } \\
\cline { 2 - 4 } & $35-49$ & $50-64$ & $65-79$ \\
\hline Men
\end{tabular}

\begin{tabular}{llll}
\hline No treatment & 320 & 325 & 283
\end{tabular}

Risk group:

\begin{tabular}{lccc}
\hline$<5 \%$ & $263(82)$ & $71(22)$ & 0 \\
\hline $5 \%-9 \%$ & $42(13)$ & $124(38)$ & $14(5)$ \\
\hline $10 \%-14 \%$ & $5(2)$ & $55(17)$ & $54(19)$ \\
\hline $15 \%-19 \%$ & $4(1)$ & $36(11)$ & $65(23)$ \\
\hline$\geqslant 20 \%$ & $6(2)$ & $39(12)$ & $150(53)$ \\
\hline Mean risk (\% risk next 5 years) & 3.4 & 10.3 & 19.6 \\
\hline Treatment & 14 & 49 & 103
\end{tabular}

\begin{tabular}{|c|c|c|c|}
\hline \\
\hline \multicolumn{4}{|l|}{ Risk group: } \\
\hline$<5 \%$ & $7(50)$ & 0 & 0 \\
\hline $5 \%-9 \%$ & $6(43)$ & $8(16)$ & $1(1)$ \\
\hline $10 \%-14 \%$ & 0 & $21(43)$ & $8(8)$ \\
\hline $15 \%-19 \%$ & 0 & $7(14)$ & $11(11)$ \\
\hline$\geqslant 20 \%$ & $1(7)$ & $13(27)$ & $83(80)$ \\
\hline Mean risk (\% risk next 5 years) & 4.9 & 14.3 & 23.6 \\
\hline \multicolumn{4}{|l|}{ Women } \\
\hline No treatment & 315 & 293 & 258 \\
\hline \multicolumn{4}{|l|}{ Risk group: } \\
\hline$<5 \%$ & $309(98)$ & $190(65)$ & $31(12)$ \\
\hline $5 \%-9 \%$ & $6(2)$ & $67(23)$ & $90(35)$ \\
\hline $10 \%-14 \%$ & 0 & $21(7)$ & $67(26)$ \\
\hline $15 \%-19 \%$ & 0 & $6(2)$ & $29(11)$ \\
\hline$\geqslant 20 \%$ & 0 & $9(3)$ & $41(16)$ \\
\hline Mean risk (\% risk next 5 years) & 1.1 & 5.4 & 11.7 \\
\hline Treatment & 7 & 60 & 131 \\
\hline \multicolumn{4}{|l|}{ Risk group: } \\
\hline$<5 \%$ & $6(86)$ & $10(17)$ & 0 \\
\hline $5 \%-9 \%$ & $1(14)$ & $22(37)$ & $16(12)$ \\
\hline $10 \%-14 \%$ & 0 & $13(21)$ & $31(24)$ \\
\hline $15 \%-19 \%$ & 0 & $7(12)$ & $21(16)$ \\
\hline$\geqslant 20 \%$ & 0 & $8(13)$ & $63(48)$ \\
\hline Mean risk (\% risk next 5 years) & 2.0 & 9.6 & 16.9 \\
\hline
\end{tabular}


Table 3 Influence of different treatment thresholds on number of people for whom treatment is recommended and number of disease events averted. Values are numbers (percentages); $\mathrm{n}$ is the number of Auckland residents in each age group

\begin{tabular}{|c|c|c|c|c|}
\hline & \multicolumn{3}{|c|}{ Age group (years) } & \multirow[b]{2}{*}{ Total } \\
\hline & $\begin{array}{c}35-49 \\
(\mathrm{n}=195696)\end{array}$ & $\begin{array}{c}50-64 \\
(n=117 \text { 132) }\end{array}$ & $\begin{array}{c}65-79 \\
(n=77 \text { 664) }\end{array}$ & \\
\hline \multicolumn{5}{|l|}{ Baseline case (no drug treatment) } \\
\hline Disease events in 5 years if no treatment & 4360 & 9751 & 13257 & 27368 \\
\hline \multicolumn{5}{|l|}{ Current guidelines for drug treatment ${ }^{\star}$} \\
\hline People currently treated & $6080(3.1)$ & $17246(14.7)$ & $23048(29.7)$ & $46374(11.9)$ \\
\hline Disease events in 5 years & 4297 & 9241 & 12141 & 25679 \\
\hline Events averted compared with no treatment & $63(1.4)$ & $510(5.2)$ & $1116(8.4)$ & $1689(6.2)$ \\
\hline \multicolumn{5}{|l|}{$10 \%$ threshold for drug treatment $\dagger$} \\
\hline People who would stop treatment & $5519(2.8)$ & $5942(5.1)$ & $1760(2.3)$ & $13221(3.4)$ \\
\hline People who would start treatment & $3457(1.8)$ & $12760(10.9)$ & 16405 (21.1) & $32622(8.4)$ \\
\hline People recommended for treatment & $4018(2.1)$ & $24064(20.5)$ & $37693(48.5)$ & $65775(16.8)$ \\
\hline Overall change in number eligible for treatment & $-2062(-1.1)$ & $6818(5.8)$ & $14645(18.9)$ & $19401(5.0)$ \\
\hline Disease events caused by stopping treatment & 58 & 119 & 48 & 225 \\
\hline Disease events averted by starting treatment & 70 & 442 & 852 & 1364 \\
\hline Overall reduction in disease events & $12(0.3)$ & $323(3.5)$ & $804(6.6)$ & $1139(4.4)$ \\
\hline \multicolumn{5}{|l|}{$15 \%$ threshold for drug treatment $\dagger$} \\
\hline People who would stop treatment & $5519(2.8)$ & $9464(8.1)$ & $5356(6.9)$ & $20339(5.2)$ \\
\hline People who would start treatment & $3157(1.6)$ & $8765(7.5)$ & $13014(16.8)$ & $24936(6.4)$ \\
\hline People recommended for treatment & $3718(1.9)$ & $16547(14.1)$ & 30706 (39.5) & $50971(13.1)$ \\
\hline Overall change in number eligible for treatment & $-2362(-1.2)$ & $-699(-0.6)$ & $7658(9.9)$ & $4597(1.2)$ \\
\hline Disease events caused by stopping treatment & 58 & 252 & 199 & 509 \\
\hline Disease events averted by starting treatment & 68 & 320 & 741 & 1129 \\
\hline Overall reduction in disease events & $10(0.2)$ & $68(0.7)$ & $542(4.5)$ & $620(2.4)$ \\
\hline \multicolumn{5}{|l|}{$20 \%$ threshold for drug treatment $\dagger$} \\
\hline People who would stop treatment & $5519(2.8)$ & $11471(9.8)$ & $7078(9.1)$ & $24068(6.2)$ \\
\hline People who would start treatment & $2864(1.5)$ & $6333(5.4)$ & $10156(13.1)$ & $19353(5.0)$ \\
\hline People recommended for treatment & $3425(1.8)$ & $12108(10.3)$ & $26126(33.6)$ & $41659(10.7)$ \\
\hline Overall change in number eligible for treatment & $-2655(-1.4)$ & $-5138(-4.4)$ & $3078(4.0)$ & $-4715(-1.2)$ \\
\hline Disease events caused by stopping treatment & 58 & 345 & 300 & 703 \\
\hline Disease events averted by starting treatment & 64 & 229 & 614 & 907 \\
\hline Overall reduction in disease events & $6(0.1)$ & $-116(-1.3)$ & $314(2.6)$ & $204(0.8)$ \\
\hline
\end{tabular}

${ }^{*}$ Compared with no treatment.

†Compared with current treatment.

ment but treatment would be recommended for 24936 patients who were not being treated. Using this threshold, it was predicted that a net increase of 620 events would be averted over 5 years. This predicted $37 \%$ increase in events averted would only require that 4597 additional people be treated (about 8000 more men and 3500 fewer women), representing an increase of less than $10 \%$ in the number treated.

Setting the threshold for treatment at a 5 year risk of disease of $20 \%$ leads to about a $10 \%$ net reduction in patients for whom drug treatment would be recommended; this represents about 4715 people (about 2500 more men and 7000 fewer women). However, this would still avert almost 200 additional disease events over 5 years compared with current treatment criteria. Using the $20 \%$ threshold would increase the number being treated only among 65-79 year olds (by 3078), leading to 314 fewer disease events. A large fall in the number treated (of 5138) and a rise in the number of disease events (by about 116) was predicted to occur among 50-64 year olds

\section{Discussion}

\section{Principal findings}

This study shows that using the absolute risk of cardiovascular disease to set thresholds for drug treatment of hypertension in primary care could significantly improve the efficiency of the management of risk. In this study, most patients for whom new treatment would be recommended, and who would therefore receive the benefits of treatment, were older than 65 . This is the age group that is most likely to be defined as undertreated when treatment thresholds that use absolute risk are applied. Implementing a 10\% threshold for a 5 year risk of cardiovascular disease would increase the proportion of patients recommended for treatment by about two fifths and the proportion of events averted by two thirds. A 15\% threshold of 5 year risk would have a minimal net impact on the proportion of people for whom treatment is recommended (an increase of $<10 \%$ ) but was predicted to avert one third more events than current criteria for treatment. The more conservative 20\% threshold would reduce the proportion of people for whom treatment is recommended by about $10 \%$ and yet still avert $10 \%$ more events than current criteria.

\section{Strengths and weaknesses of the study}

A strength of this study was that data on risk factors for cardiovascular disease were available for a large, representative sample of adults who were middle aged and older in an urban population that accounts for more than one quarter of all New Zealanders. Although the response rate was only $72 \%$, investigations of non-responders in prevalence studies of cardiovascular risk factors have shown that non- 
response rates of one third bias prevalence estimates by less than $5 \%{ }^{17}$

Participants' blood pressure was estimated from only two measurements taken at one sitting, which may overestimate average blood pressure, although the measurements were made in an environment that should not have been stressful. The results of only one measurement of blood lipids were available; most treatment guidelines recommend evaluating at least two before making treatment decisions. The risk equation used did not include a family history of cardiovascular disease or other risk factors for cardiovascular disease, such as obesity, nor did it include protective factors, such as physical activity.

The prediction equation from the Framingham study used here has been validated in a number of similar populations ${ }^{18-21}$; we were able to predict current national mortality from cardiovascular disease in New Zealand with reasonable accuracy (data not shown). The $25 \%$ estimate of the relative effect of drug treatment is conservative and is based on evidence from randomised controlled trials. ${ }^{122}{ }^{23}$ A series of sensitivity analyses were undertaken in this study, including varying the increment of change in blood pressure for those already receiving treatment from 0 to $12 \mathrm{~mm} \mathrm{Hg}$ systolic and $6 \mathrm{~mm} \mathrm{Hg}$ diastolic and varying the relative benefit of treatment from one quarter to one third. Neither of these analyses had a substantive effect on the results.

It is unlikely that patients receiving treatment who have low estimated risks of cardiovascular disease would have their treatment withdrawn. Therefore, the benefits of treatment criteria based on absolute risk would apply to future groups of patients rather than current groups. Confidence intervals around the estimated risks and benefits were not calculated because of the multiple assumptions made in the analyses. The study did not examine people older than 80 because the Framingham equation does not predict risk in the very old. However, there is increasing evidence from randomised trials that people older than 80 benefit from treatment to lower their blood pressure. $^{24}$ There are a family of Framingham equations for predicting different outcomes. These are all highly correlated. Sensitivity analysis using different endpoints gives the same pattern of findings but different absolute event rates.

We are unaware of any published studies that have applied recommendations for managing blood pressure that are based on the absolute risk to each individual in a representative population sample using a comprehensive prediction model based on the absolute risk of disease. Previous studies have examined only patients being treated or have tended to apply simplified equations of risk prediction to groups of people rather than to individuals. ${ }^{25-27}$

\section{Implications for clinicians and policymakers}

This analysis may be helpful to those who develop guidelines for managing blood pressure when deciding on recommendations for treatment thresholds. For example, if the New Zealand health services were unable to allocate significantly more resources for managing hypertension then a 5 year threshold of a $15 \%$ risk would be almost cost neutral yet would increase the number of events averted by up to one third. Using the lower 5 year threshold of a $10 \%$ risk, which is the basis of current recommendations, would lead to a significant increase in treatment if fully implemented; the higher $20 \%$ threshold would reduce current levels of treatment. Additional approaches to improving the cost effectiveness of treatment include substituting cheaper but proven alternative treatments, such as diuretics and $\beta$ blockers, for expensive newer drugs.

At the middle threshold ( 5 year risk of $15 \%$ ), about 20 patients would require drug treatment for 5 years to avert one disease event. Before lowering the threshold for mass treatment below this, it would seem reasonable to identify and treat more systematically patients who are at highest risk. The most neglected groups are the elderly and the 5\%-10\% of people with a history of cardiovascular disease who are at very high risk and account for more than one third of all disease events in Auckland (unpublished data).

\section{Unanswered questions}

Thresholds based on absolute risk favour treatment of the elderly and other high risk groups at the expense of younger people at lower risk, especially women. This study did not take account of the greater number of potential years of life gained by younger people who benefit from having an event averted. A useful extension to the analyses would examine the implications of applying thresholds based on potential life years gained and the quality adjusted life years gained as a result of treatment. However, in a context in which many practitioners still use blood pressure as the key determinant for treatment decisions, establishing the concept of thresholds based on absolute risk will be an important advance. Moreover, there are complexities and controversies in estimating potential life years gained or quality adjusted life years gained, such as the appropriate weighting of non-fatal events, which are more common in younger people, and the appropriateness of discounting life years gained, which can have a significant influence on age related benefits.

Many national and international guidelines on the management of raised blood pressure and other risk factors for cardiovascular disease emphasise that the absolute risk of disease is a key determinant of the need for treatment. Moreover, a number, including those from the British Hypertension Society ${ }^{4}$ and the Joint British and Joint European Societies, ${ }^{56}$ have followed New Zealand's lead of recommending quantitative treatment thresholds based on absolute risk. ${ }^{8}$ Although estimating the absolute risk of cardiovascular disease has been simplified by the production of risk charts $^{35}{ }^{510}$ and computer programs, ${ }^{2728}$ practitioners in primary care will require considerable support if they are to systematically implement management guidelines based on risk. Computer based systems for disease and risk management are being used increasingly in primary care and will make estimating the risk of multiple factors much easier, once these factors have been electronically stored in a patient's record.

\section{Conclusion}

This study highlights the potential for improving the prevention of cardiovascular disease in primary care by using treatment thresholds that are based on absolute 


\section{What is already known on this topic}

Numerous trials have shown that treatment to lower blood pressure significantly reduces the relative risk of cardiovascular disease in all patients with hypertension

The absolute benefits of treatment depend primarily on the absolute risks of cardiovascular disease that exist before treatment

\section{What this study adds}

In this study the absolute benefits of treatment to lower blood pressure are estimated for current practice and for three different thresholds of treatment based on absolute risk of cardiovascular disease

Targeting patients at high risk of cardiovascular disease is likely to significantly improve the efficiency of treatment to lower blood pressure

risk for managing raised blood pressure. The study also illustrates the comparatively simple analyses that guideline developers can use to investigate the likely impact of their recommendations on patients and populations.

Contributors: RJ designed the original prevalence survey, supervised $\mathrm{PP}$ and $\mathrm{SB}$, and will act as guarantor for the paper. PP managed the prevalence survey and initiated the modelling analyses. SB further developed and refined the modelling, undertook the analyses, and wrote the paper.

Funding: The study on which these analyses were based was funded by the National Heart Foundation of New Zealand and the Health Research Council of New Zealand. The work of SB and PP was partly funded by the New Zealand Committee of the Australasian Faculty of Public Health Medicine.

Competing interests: None declared.

1 Gueyffier F, Boutitie F, Boissel J-P, Pocock S, Coope J, Cutler J, et al. Effect of antihypertensive drug treatment on cardiovascular outcomes in women and men: a meta-analysis of individual patient data from randomised controlled trials. Ann Intern Med 1997;126:761-7.

2 MacMahon S, Rodgers A. The effects of antihypertensive treatment on vascular disease: reappraisal of the evidence in 1994. J Vasc Med Biol 1993;4:265-71.

3 Core Services Committee. Guidelines for the management of raised blood pressure in New Zealand. Wellington: Ministry of Health, 1995.

4 Ramsay LE, Williams B, Johnston GD, MacGregor GA, Poston L, Potter JF, et al. British Hypertension Society guidelines for hypertension management 1999: summary. BMJ 1999;319:630-5.

5 Joint British recommendations on prevention of coronary heart disease in clinical practice. Heart 1998;80(suppl 2):1-29S.

6 Wood D, De Backer G, Faergeman O, Graham I, Mancia G, Pyorala K. Prevention of coronary heart disease in clinical practice: recommenda- tions of the Second Joint Task Force of European and other Societies on Coronary Prevention. Atherosclerosis 1998;140:199-270.

7 Anderson K, Odell P, Wilson P, Kannel W. Risk profiles. Am Heart J 1991;121:293-8.

8 Jackson R, Barham P, Bills J, Birch T, McLennan L, MacMahon S, et al. The management of raised blood pressure in New Zealand: a discussion document. BMJ 1993;307:107-10.

9 Mann J, Crooke M, Fear H, Hay D, Jackson R, Neutze J, et al. Guidelines for detection and management of dyslipidaemia. $N$ Z Med J 1993;106:133-42.

10 Dyslipidaemia Advisory Group. 1996 National Heart Foundation clinical guidelines for the assessment and management of dyslipidaemia. $N Z$ Med J 1996;109:224-31.

11 North D, Priest P, Lay-Yee R, Jackson R. New Zealand guidelines for the management of dyslipidaemia: implications for treatment in an urban New Zealand population. N Z Med J 1996;109:134-7.

12 Allain C, Poon L, Chan C. Enzymatic determination of total serum cholesterol. Clin Chem 1973;20:474-5.

13 Lopes-Virella M. Cholesterol determination in high density lipoproteins separated by three different methods. Clin Chemistry 1977;23:882-4.

14 Scandinavian Simvastatin Survival Study Group. Randomised trial of cholesterol lowering in 4444 patients with coronary heart disease: the Scandinavian simvastatin survival study. Lancet 1994;344:1383-9.

15 Sacks F, Pfeffer M, Moye L, Rouleau J, Rutherford J, Cole T, et al. The effect of pravastatin on coronary events after myocardial infarction in patients with average cholesterol levels. $N$ Engl J Med 1996;335:1001-9.

16 The Long-term Intervention with Pravastatin in Ischaemic Disease (LIPID) Study Group. Prevention of cardiovascular events and death with pravastatin in patients with coronary heart disease and a broad range of initial cholesterol levels. N Engl J Med 1998;339:1349-57.

17 Jackson R, Chambless L, Yang K, Byrne T, Watson R, Folsom A, et al. Differences between respondents and nonrespondents in a multi-center community-based study vary by age and ethnicity. J Clin Epidemiol 1996;49:1441-6

18 Leaverton P, Sorlie P, Kleinman J, Dannenberg A, Ingster-Moore L, Kannel W, et al. Representativeness of the Framingham risk model for CHD mortality: a comparison with a national cohort. J Chronic Dis 1987;40:775-84.

19 Brand R, Rosenman R, Sholtz R, Friedman M. Multivariate prediction of coronary heart disease in the Western collaborative group study compared to the findings of the Framingham Study. Circulation 1976;53:348-55.

20 McGee D, Gordon T. The results of the Framingham study applied to four other US-based epidemiologic studies of cardiovascular disease. In: Kannel W, Gordon T, editors. The Framingham study: an epidemiological investigation of cardiovascular disease. Bethesda, MD: US Department of Health, Education, and Welfare, 1976.

$21 \mathrm{Haq}$ I, Yeo W, Ramsay L, Jackson P. Is the Framingham risk function valid for northern European populations? A comparison of methods for estimating acute risk in high risk men. Heart 1999;81:40-6.

22 Insua J, Sacks H, Lau T, Lau J, Reitman D, Pagano D, et al. Drug treatment of hypertension in the elderly: a meta-analysis. Ann Intern Med $1994 ; 121: 355-62$.

23 Mulrow C, Cornell J, Herrera C, Kadri A, Farnett L, Aguilar C. Hypertension in the elderly: implications and generalisability of randomised trials. JAMA 1994:272:1932-38.

24 Gueyffier F, Bulpitt C, Boissel J-P, Schron E, Ekbom T, Fagard E, et al. Antihypertensive drugs in very old people: a subgroup meta-analysis of randomised controlled trials. Lancet 1999;353:793-6.

25 Fahey T, Peters T. What constitutes controlled hypertension? Patien based comparison of hypertension guidelines. BMJ 1996;313:93-6.

26 Johannesson M. The impact of age on the cost-effectiveness of hypertension treatment: an analysis of randomised drug trials. Med Decis hypertension treatmen : a

27 Hingorani A, Vallance P. A simple computer program for guiding management of cardiovascular risk factors and prescribing. $B M J$ 1998;318:101-5.

28 New Zealand Guideline Group. Management of raised blood pressure in New Zealand (appendix 4). www.nzgg.org.nz/library/gl_complete/ bloodpressure (accessed 25 February 2000).

(Accepted 14 February 2000)

\section{One hundred years ago Discovery of a Temple of Esculapius}

A discovery was recently made at Stankeny, in the island of Cos, which, it is hoped, will lead to the bringing to light of the famous Temple of Æsculapius. On April 4th a Mussulman labourer who was planting a vine struck with his spade a stone cupola. He at once notified the authorities, and excavations were undertaken under the direction of a Kaimakan, which exposed a mausoleum of 30 to 45 square metres in area, in which were a number of bones and fragments of pottery. The monument, which is built of brick, appears to date from the Roman period; a ladder 2 to 3 metres in length leads down into the interior, which has the appearance of a chapel. Opposite the entrance are three niches, each capable of containing a coffin, above there is an opening in the form of a low door, and on each side are two other niches. No inscription has been discovered, but there are certain indications which in the eyes of local archæologists give ground for the supposition that in the immediate neighbourhood there was a temple or some other public establishment, and it is thought not improbable that it may be the Temple of Esculapius.

(BMJ 1900;i:1112.) 